\title{
Lettres de Jean-Luc Raharimanana à Serge Meitinger (1987-1989) avec quelques poèmes
}

\section{Serge Meitinger}

\section{OpenEdition}

\section{Journals}

Electronic version

URL: http://journals.openedition.org/coma/650

DOI: $10.4000 /$ coma.650

ISSN: 2275-1742

\section{Publisher}

Institut des textes \& manuscrits modernes (ITEM)

\section{Electronic reference}

Serge Meitinger, « Lettres de Jean-Luc Raharimanana à Serge Meitinger (1987-1989) avec quelques poèmes », Continents manuscrits [Online], 6 | 2016, Online since 31 October 2016, connection on 01 May 2019. URL : http://journals.openedition.org/coma/650 ; DOI : 10.4000/coma.650

This text was automatically generated on 1 May 2019.

\section{(c)}

Continents manuscrits - Génétique des textes littéraires - Afrique, Caraîbe, dispora est mis à disposition selon les termes de la licence Creative Commons Attribution - Pas d'Utilisation

Commerciale - Pas de Modification 4.0 International. 


\title{
Lettres de Jean-Luc Raharimanana à Serge Meitinger (1987-1989) avec quelques poèmes
}

\author{
Serge Meitinger
}

1 En vue de la composition de ce numéro de Continents manuscrits, je suis revenu à mes archives où j'ai retrouvé une série de lettres et de poèmes de Jean-Luc Raharimanana qu'il m'adressa entre 1987 et 1989 à la suite de notre rencontre lors du colloque «Jean-Joseph Rabearivelo, cet inconnu?» (université d'Antananarivo, faculté des Lettres et Sciences Humaines, mai 1987) et de la remise du prix littéraire décerné sous le nom de Rabearivelo à cette occasion, prix qu'il partagea avec David Jaomanoro (qui était mon étudiant à l'École Normale, niveau III). J'ai eu envie de savoir si mes lettres avaient pu être conservées et j'ai écrit à l'auteur qui, après enquête, m'a répondu ceci, par le moyen de courrier actuel le plus répandu, le mail :

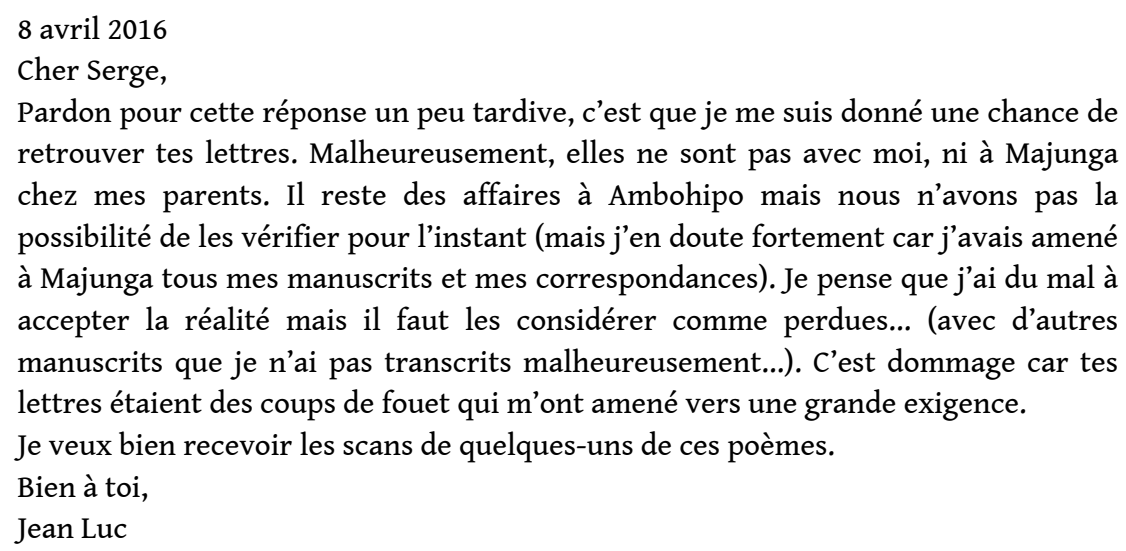

2 Cette perte n'enlève rien aux documents qui restent. Je me rappelle que j'avais réagi avec intérêt mais aussi avec sévérité aux envois reçus à la suite de notre prise de contact. Avec une première lettre en juin, j'avais trouvé une série de poèmes intitulée « Le poème des sensations » composée de neuf textes. J'avais demandé au jeune poète de corriger des 
fautes de langue et des impropriétés, signalant que ces scories ne pourraient que le desservir auprès du public et des éditeurs potentiels. Je l'incitais également à se cultiver par la lecture des grands auteurs et même des critiques, à se familiariser avec l'histoire de la littérature, française bien sûr mais pas seulement car il y a une vraie internationale des écrivains qui trouvent leur bien sur toute la planète (Rabearivelo a montré l'exemple sur ce point). En octobre de la même année, une lettre accompagnait «Au plus près de l'éloigné ", série de cinq poèmes assez longs qui m'apparurent comme une incitation un peu bavarde parfois à l'entrée dans une vie nouvelle envisagée sur le fond d'un mouvement cosmique. En voici l'ultime feuillet :

Fig. 1 : JLR, Au plus près de l'éloigné, $V$

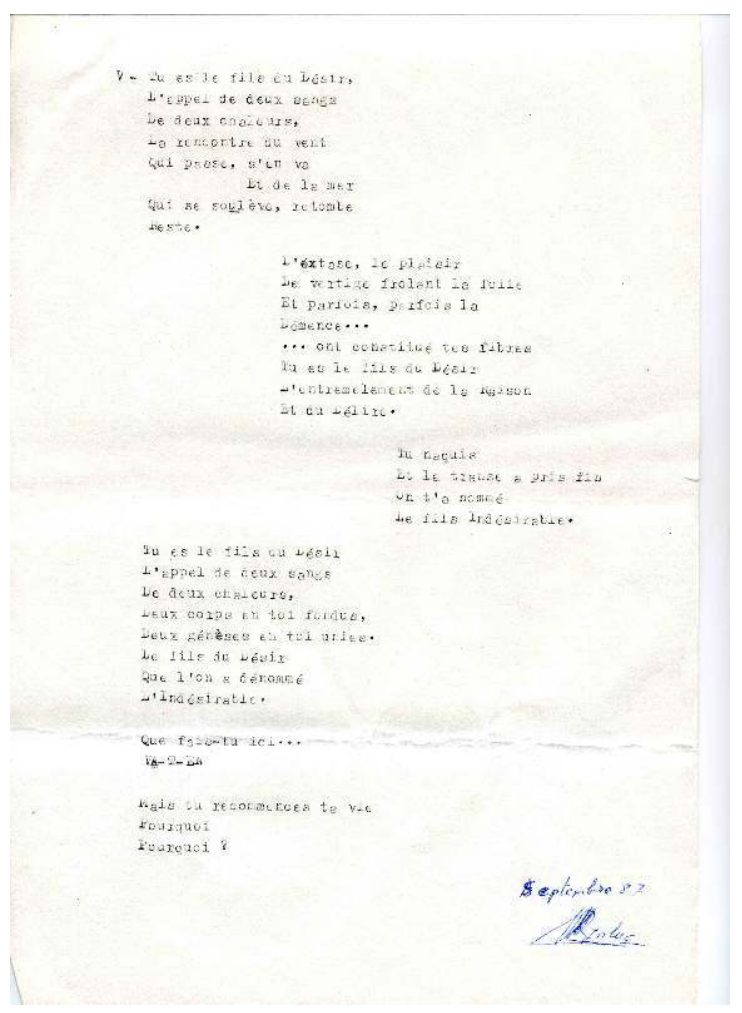

Archive personnelle de Serge Meitinger 
Transcription d'Au plus près de l'éloigné, $V$

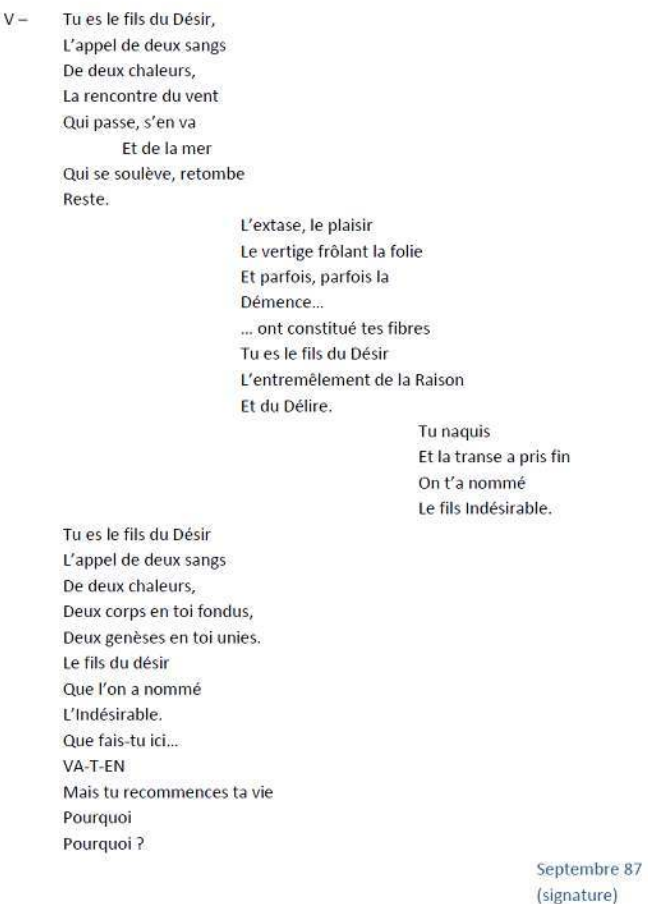

3 Il y eut aussi une autre séquence de dix poèmes, sans titre d'ensemble, dont je ne sais plus s'ils me sont parvenus avec la lettre d'octobre ou celle de décembre. En voici une page : 
Fig. 2 : JLR, Poème, 5 : Voyage

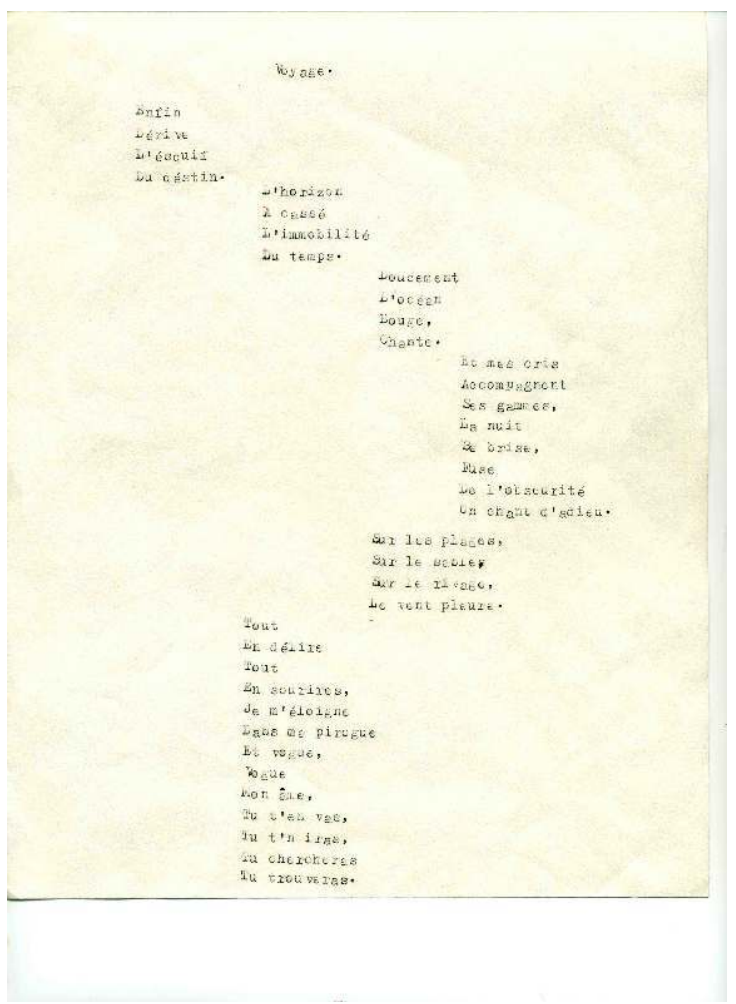

Archive personnelle de Serge Meitinger

Transcription du Poème 5 : Voyage

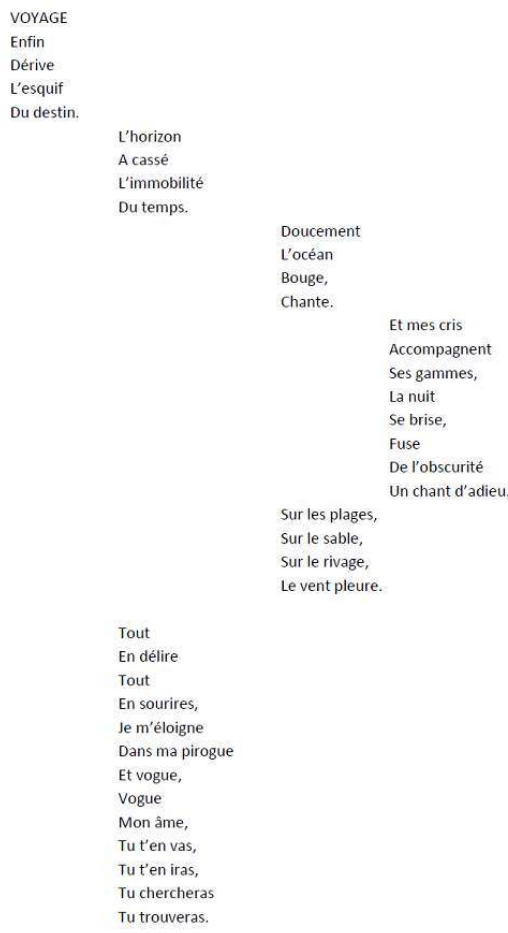


4 La lettre de décembre 1987 réagissait avec quelque vigueur à mes remarques critiques et se voulait un plaidoyer pour la sincérité et déjà l'authenticité.

Fig. 3 : JLR, lettre à Serge Meitinger du 14 décembre $1987 \mathrm{f}^{\circ} 1 \mathrm{r}$.

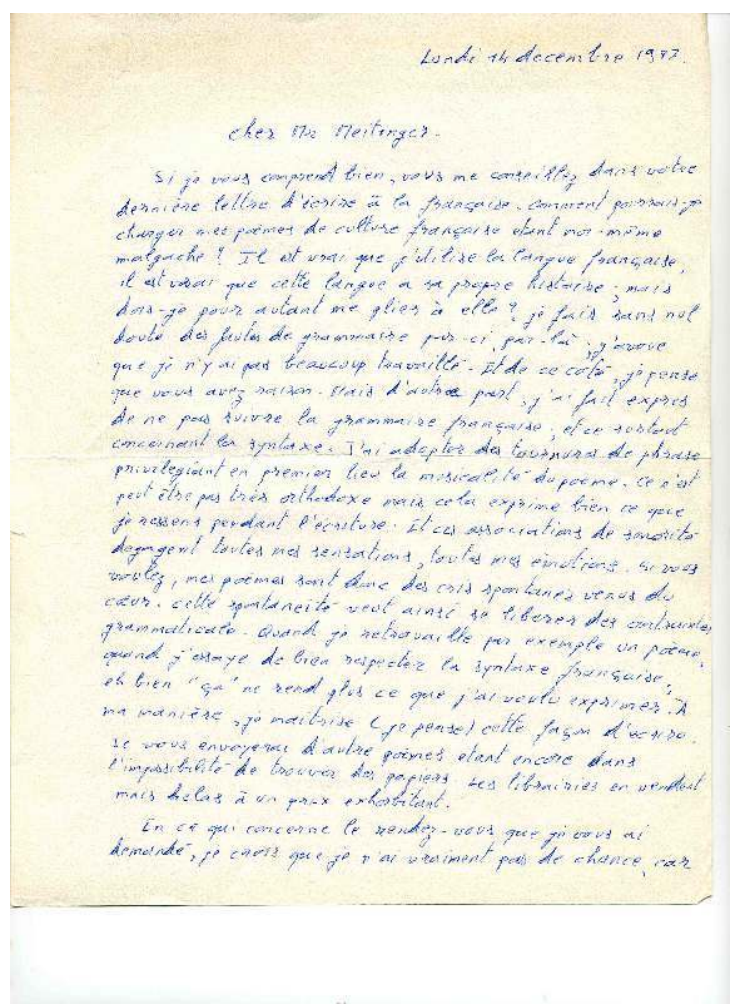

Archive personnelle de Serge Meitinger 
Fig. 4 : JLR, Lettre à Serge Meitinger du 14 décembre $1987 \mathrm{f}^{\circ} 1 \mathrm{v}$.

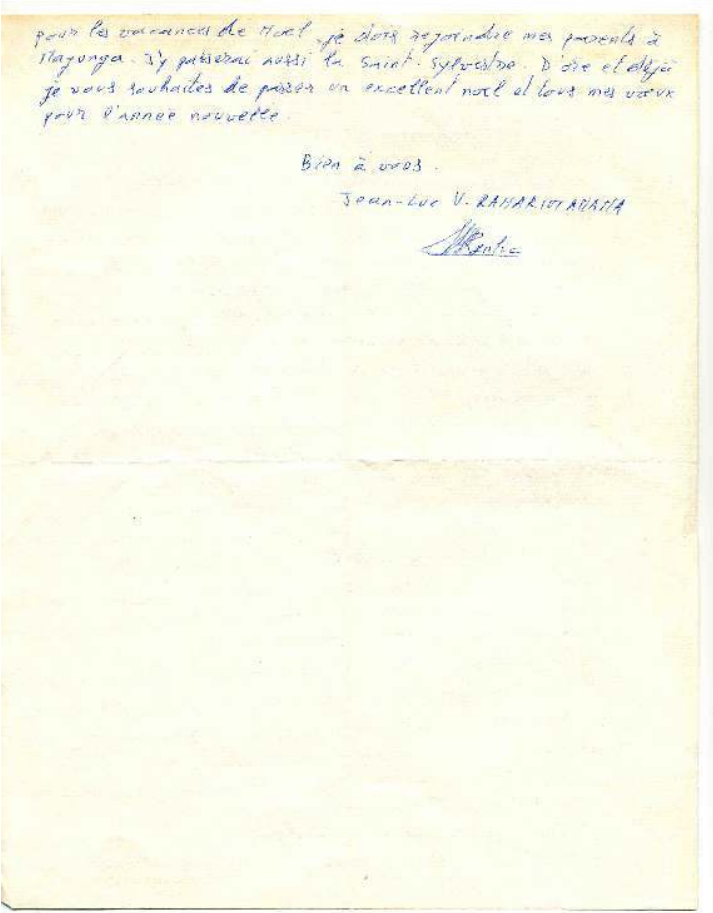

Archive personnelle de Serge Meitinger

Saisie de la lettre du 14 décembre 1987 (fig. 3 et 4)

Cher Mr Meitinger,

Si je vous comprend (sic) bien, vous me conseillez dans votre dernière lettre d'écrire à la française. Comment pourrais-je charger mes poèmes de culture française étant moi-même malgache? II est vrai que j'utilise la langue française, il est vrai que cette langue a sa propre histoire, mais dois-je pour autant me plier à elle ? je fais sans nul doute des fautes de grammaire par-ci par-là ; j'avoue que je n'y ai pas beaucoup travaillé. Et de ce côté, je pense que vous avez raison. Mais d'autre part, j'ai fait exprès de ne pas suivre la grammaire française ; et ce surtout concernant la syntaxe. J'ai adopter (sic) des tournures de phrase privilégiant en premier lieu la musicalité du poème. Ce n'est peut-être pas très orthodoxe mais cela exprime bien ce que je ressens pendant l'écriture. Et ces associations de sonorité dégagent toutes mes sensations, toutes mes émotions. Si vous voulez, mes poèmes sont donc des cris spontanés venus du cœur. Cette spontanéité veut ainsi se libérer des contraintes grammaticales. Quand je retravaille par exemple un poème, quand j'essaye de bien respecter la syntaxe française, eh bien " ça » ne rend plus ce que j'ai voulu exprimer. A ma manière, je maîtrise (je pense) cette façon d'écrire. Je vous envoyerai (sic) d'autres poèmes étant encore dans l'impossibilité de trouver des papiers. Les librairies en vendent mais hélas à un prix exorbitant.

En ce qui concerne le rendez-vous que je vous ai demandé, je crois que je n'ai vraiment pas de chance, car pour les vacances de Noël, je dois rejoindre mes parents à Majunga. J'y passerai aussi la Saint-Sylvestre. D'ore et déjà je vous souhaite de passer un excellent Noël et tous mes vœux pour l'année nouvelle.

Bien à vous,

Jean-LUC V. RAHARIMANANA (signature) 
5 En mars 1988, une autre lettre fait le point sur une situation d'époque ou générationnelle avec une lucidité qui explique déjà certains des choix futurs.

Fig. 5 : JLR, lettre à Serge Meitinger, du 6 mars 1988

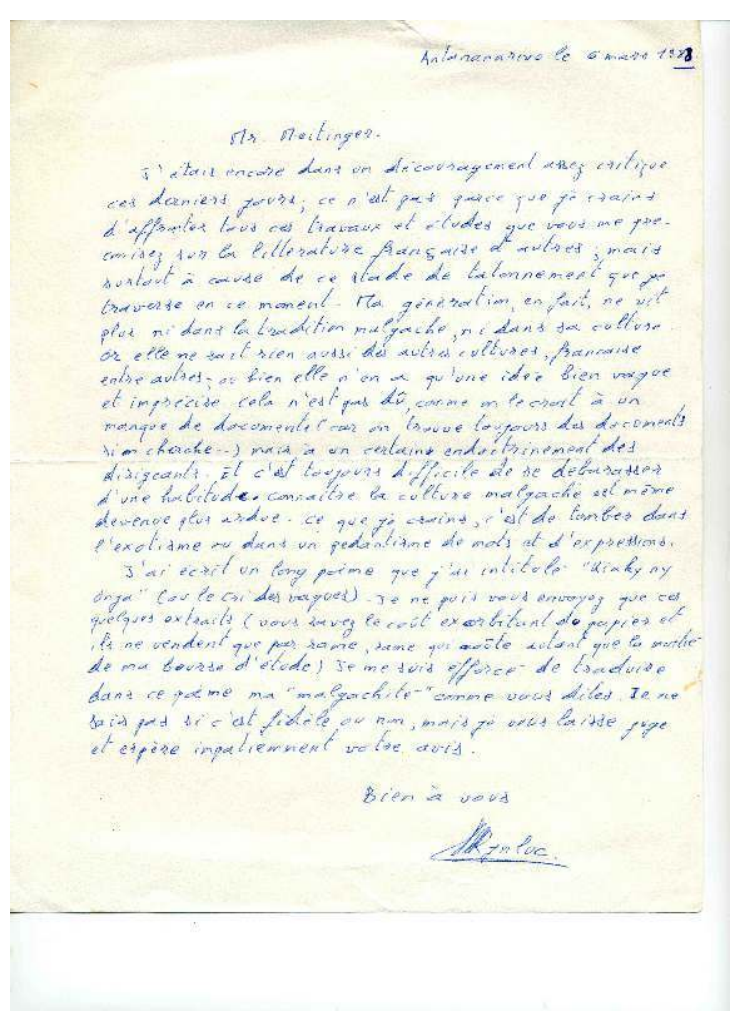

Archive personnelle de Serge Meitinger 
Transcription de la lettre du 6 mars 1988 (fig. 5)

Antananarivo, le 6 mars 1988

Mr Meitinger,

J'étais encore dans un découragement assez critique ces derniers jours; ce n'est pas parce que je crains d'affronter tous ces travaux et études que vous me préconisez sur la littérature française et autres; mais surtout à cause de ce stade de tâtonnement que je traverse en ce moment. Ma génération, en fait, ne vit plus ni dans la tradition malgache, ni dans sa culture. Or elle ne sait rien aussi des autres cultures, française entre autres ; ou bien elle n'en a qu'une idée bien vague et imprécise. Cela n'est pas dû, comme on le croit, à un manque de documents (car on trouve toujours des documents si on cherche...) mais à un certain endoctrinement des dirigeants. Et c'est toujours difficile de se débarrasser d'une habitude. Connaitre la culture malgache est même devenue plus ardue (sic). Ce que je crains, c'est de tomber dans l'exotisme ou dans un pédantisme de mots et d'expressions.

J'ai écrit un long poème que j'ai intitulé Kiaky ny onja (ou le cri des vagues). Je ne puis vous envoyer que ces quelques extraits (vous savez le coût exorbitant du papier et ils ne vendent que par rame, rame qui coûte autant que la moitié de ma bourse d'étude). Je me suis efforcé de traduire dans ce poème ma " malgachité " comme vous dites. Je ne sais pas si c'est fidèle ou non, mais je vous laisse juge et espère impatiemment votre avis.

Bien à vous

(signature)

J'ai regretté que le prix exorbitant du papier m'ait empêché d'avoir sous la main l'ensemble de ces « Cris des vagues ». Je n'en reçus que trois poèmes dont je détache celuici qui sonne plus malgache que les précédents, dans la lignée des hain-teny, de Rabearivelo et des contes où figure Zatovo. 
Fig. 6 JLR, Kiaky Ny Onja (Le cri des vagues), 9

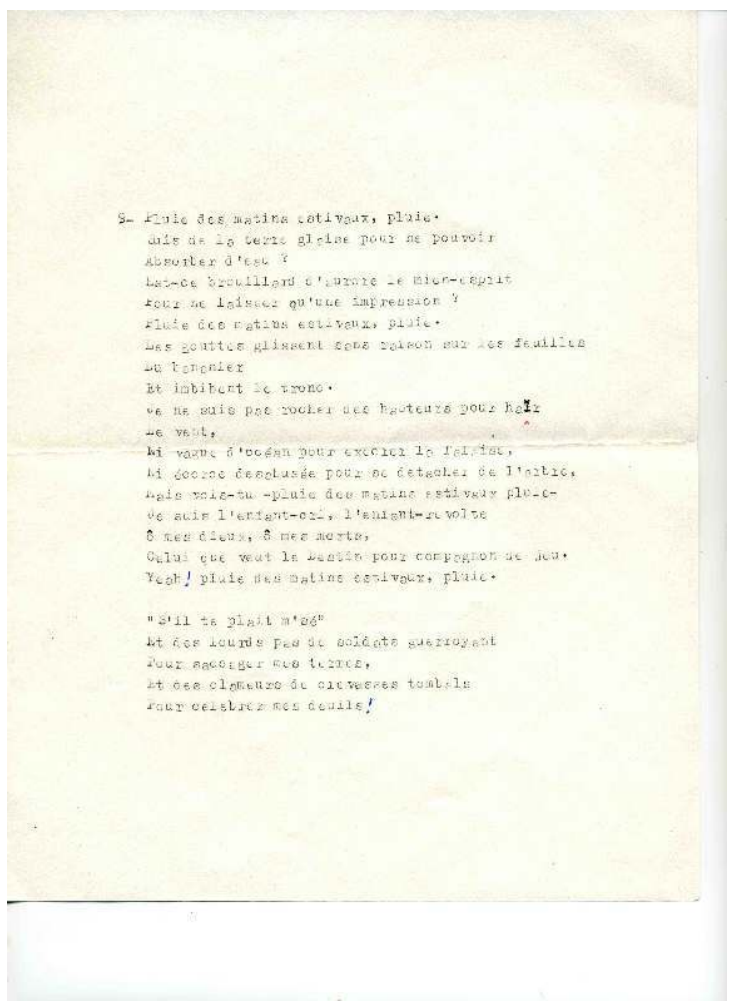

Archive personnelle de Serge Meitinger

Transcription de Kiaky Ny Onja (Le cri des vagues), 9

9. Pluie des matins estivaux, pluie.

Suis de la terre glaise pour ne pouvoir

Absorber l'eau?

Est-ce brouillard d'aurore le mien-esprit

Pour ne laisser qu'une impression?

Pluie des matins estivaux, pluie.

Des gouttes glissent sans raison sur les feuilles

Du bananier

Et imbibent le tronc.

Je ne suis pas rocher des hauteurs pour haïr

Le vent,

Ni vague d'océan pour exécrer la falaise,

Ni écorce désabusée pour se détacher de l'arbre,

Mais vois-tu - pluie des matins estivaux, pluie -

Je suis l'enfant-cri, l'enfant-révolte

ô mes dieux, ô mes morts,

Celui que veut le destin pour compagnon de jeu.

Yeah ! pluie des matins estivaux, pluie.

" S'il te plaît m'sé "

Et des lourds pas de soldats guerroyant

Pour saccager mes terres,

Et des clameurs de crevasses tombal(e)s

Pour célébrer mes deuils 
6

JLR LPdS 2 Le poème des sensations, 2

JLR.LPdS. 3 Le poème des sensations, 3.

JLR.LPdS. 4 Le poème des sensations, 4.

JLR.LPdS. 5 Le poème des sensations, 5.

JLR.LPdS. 6 Le poème des sensations, 6 .

JLR.LPdS.7 Le poème des sensations, 7.

JLR.LPdS.8 Le poème des sensations, 8.

JLR.LPdS.9 Le poème des sensations, 9 (ensemble daté du 6 juin 1987).

9 JLR.1987.10.27 Lettre à Serge Meitinger du 27 octobre 1987.

JLR.APPE.I Au plus près de l'éloigné, I.

JLR.APPE.Ib Au plus près de l'éloigné, I (fin).

JLR.APPE.II Au plus près de l'éloigné, II.

JLR.APPE.III Au plus près de l'éloigné, III.

JLR.APPE.IV Au plus près de l'éloigné, IV.

JLR.APPE.V Au plus près de l'éloigné, V (ensemble daté septembre 1987).

10 JLR.P.1.Est-il Poème, 1 : Est-il ?

JLR.P.2.Savoir l'éternité Poème, 2 : Savoir l'éternité

JLR.P.3.La voix Poème, 3 : La voix

JLR.P.4.Pays d'exil Poème, 4 : Pays d'exil

JLR.P.5.Voyage Poème, 5 : Voyage

JLR.P.6.Tes chants Poème, 6 : Tes chants

JLR.P.7.Tes regards Poème, 7 : Tes regards

JLR.P.8.Fleur du matin Poème, 8 : Fleur du matin

JLR.P.9.Les valeureux Poème, $9:$ Les valeureux

JLR.P.10.Inquiétude Poème, 10 : Inquiétude

11 JLR.1987.12.14 Lettre à Serge Meitinger du 14 décembre 1987

JLR.1987.12.14v Verso de la même lettre

12 JLR.1988.01.08 Lettre à Serge Meitinger du 8 janvier 1988

13 JLR.1988.03.06 Lettre à Serge Meitinger du 6 mars 1988

JLR.KNO.4 Kiaky Ny Onja (Le cri des vagues), 4.

JLR.KNO.9 Kiaky Ny Onja, 9.

JLR.KNO.10 Kiaky Ny Onja, 10.

14 JLR.1988.04.22 Lettre à Serge Meitinger du 22 avril 1988

JLR.1988.04.22.PrixJJR Photo : remise du prix JJR à David Jaomanoro par Serge Meitinger

15 JLR.1988.05.15 Lettre à Serge Meitinger du 15 mai 1988

JLR.1988.05.15v Verso de la même lettre 
JLR.1989.Voeuxr Carte postale : Photo : maisons de terre rouge des hauts plateaux JLR.1989.Voeuxv Recto : les vœux

INDEX

Mots-clés: Jean-Luc Raharimanana, Madagascar 